\title{
EXTRACORPOREAL MEMBRANE OXYGENATION AS A METHOD TO MANAGE ACUTE CARDIOPULMONARY FAILURE AFTER EMERGENCY CORONARY ARTERY BYPASS GRAFTING
}

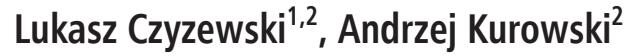 \\ 'Department of Nephrology Nursing, Medical University of Warsaw, Warsaw, Poland \\ ${ }^{2}$ Department of Anaesthesiology, Institute of Cardiology, Warsaw, Poland
}

\begin{abstract}
Acute cardiopulmonary failure after emergency coronary artery bypass grafting (CABG) is a complex therapeutic problem due to its high mortality rate. This paper reports the safety and effectiveness of extracorporeal membrane oxygenation (ECMO) in treating acute cardiopulmonary failure following emergency CABG. A 37-day period of mechanical circulation support and extracorporeal blood oxygenation with the ECMO method served as a bridge to recovery. At day 34 following the disconnection of ECMO, a patient with $\mathrm{EF}>35 \%$ was transferred to a rehabilitation ward. The procedure can be successful only when the physicians, nurses and perfusionists of the intensive care team cooperate closely.
\end{abstract}

KEY WORDS: extracorporeal membrane oxygenation, heart failure, coronary artery bypass graft

Disaster Emerg Med J 2017; (2)1: 45-49

\section{INTRODUCTION}

Acute cardiopulmonary failure is a complex therapeutic problem due to its high mortality rate. The biggest threat to the survival of a patient in acute cardiopulmonary failure is a low cardiac output, as well as the high ventilation pressures and high oxygen concentrations being used. In the clinical practice, when cardiosurgery has proved unsuccessful, the standard treatment is to apply an intensive medical treatment with potent pressure agents and intra-aortic balloon counterpulsation, (IABP) [1]. Depending on how a particular ward is equipped, the treatments available also include ECMO (Extracorporeal Membrane Oxygenation). This method, applied at the right moment, i.e. before irreversible organ changes occur, provides an opportunity to save and prolong life. Depending on which procedural strategy is chosen, ECMO can be used in one of the three following ways: as a bridge to making a decision; as a bridge to transplantation; or as a bridge to recovery [2]. There are two main types of cannulating a patient so that he/she will be connected into the ECMO system [3]: veno-venous cannulation, which is used in respiratory failure without the heart failure component; and arterio-arterial cannulation, used in respiratory failure in a patient unstable hemodynamically. The cannulation used in ECMO can either be percutaneous [2] or via an open surgical approach [4].

The subject of the paper presented here is to prove the safety and effectiveness of ECMO in treating acute cardiopulmonary failure after $C A B G$, as a bridge to recovery.

\section{CASE}

A 53-year-old man, with a negative history of cardiovascular diseases, was admitted to our hospital in 
June 2014. The diagnosis was acute coronary syndrome - inferior wall STEMI (ST elevation mycoradial information). Following emergency coronarography, an attempt was made to open the right coronary artery - the procedure was unsuccessful, complicated by the occlusion of the right coronary branch. Echocardiography revealed the following: a normal size of the left ventricle (LV); a large akinesis of the LV muscle; hypokinesis of the distal parts of the inferolateral and anterolateral walls; and substantial hypokinesis of the proximal part of the anterolateral wall. Ejection fraction (EF) was around 15\%. Despite intensive treatment, the condition of the patient gradually deteriorated - there were several episodes of paroxysmal atrial fibrillation and flutter. On the 7th day after STEMI, the patient had a sudden cardiac arrest with asystole and on two occasions ventricular fibrillation terminated with external defibrillation.

The patient underwent an off-pump coronary artery bypass graft (CABG): left internal mammary artery (LIMA) - left anterior descending artery (LAD), saphenous vein (SV) - right coronary artery (RCA). Once the patient was transferred to a post-operative ward, he underwent analgosedation and was ventilated mechanically with synchronized intermittent mandatory ventilation (SIMV) - fraction of inspired oxygen $\left(\mathrm{FiO}_{2}\right)$ was 0.6 , the circulation was stabilized with continuous infusions of dobutamine and nitroglycerin achieving RR 150/80 mm Hg, sinus rhythm $95 \mathrm{bpm}$ and spontaneous diuresis.

By the end of day 4 following CABG, the patient was woken up and fully conscious, extubated, with only passive oxygen therapy used. There were numerous episodes of ventricular fibrillation (VF) terminated by defibrillation and he regained consciousness after interventions. Antiarrhythmic treatment with amiodarone and lidocaine was performed in the form of permanent infusion. EF was around $10 \%$. A balloon for intra-aortic counterpulsation was implanted, yet no hemodynamic stability was achieved. In the face of acute cardiopulmonary failure which did not respond to standard treatment, the decision was made to apply extracorporeal arterio-venous oxygenation ECMO. A POL-VAD cannula was inserted into the right atrium, an arterial cannula was connected to the aorta. A vent was inserted into the left ventricle via the right upper pulmonary vein. In this way a very good venous outflow was established, which fully relieved the burden on the heart. The patient was under balanced anaesthesia. During the procedure there were several sudden cardiac arrests due to $V F$, which were successfully terminated by defibrillation. Once the patient was connected to ECMO, his condition became stable, which made it possible to reduce catecholamines and to balance metabolic asidosis. The patient was ventilated mechanically with $\mathrm{SIMV} \mathrm{FiO}_{2} 0.4, \mathrm{ECMO}$ — CO 5.0 achieving MABP > $90 \mathrm{~mm} \mathrm{Hg}$ (Fig. 1, 2).

On the 5th day after $C A B G$, the patient underwent reoperation because of bleeding (hct 18.6\%, $\mathrm{Hb} 6.12 \mathrm{~g} / \mathrm{dl}$ ). Sedation was achieved with an i.v. infusion of sodium thiopental. ECMO was closely monitored and maintained by a perfusionist team during the entire duration of support. The support system was monitored daily for thrombi and fibrin deposits.

Between days 6 and 11 following CABG, the patient underwent analgosedation (dexmedetomidine + sufentanil) together with protective mechan-

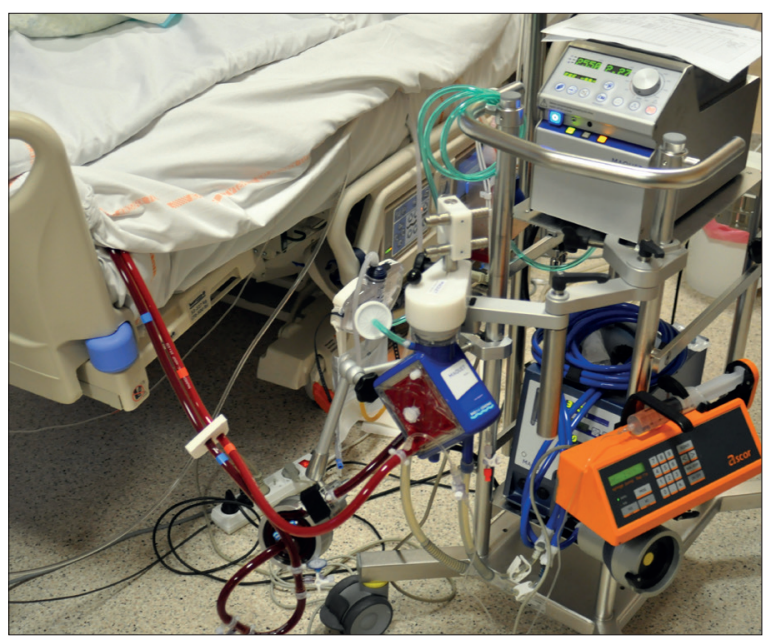

FIGURE 1. Extracorporeal Membrane Oxygenation

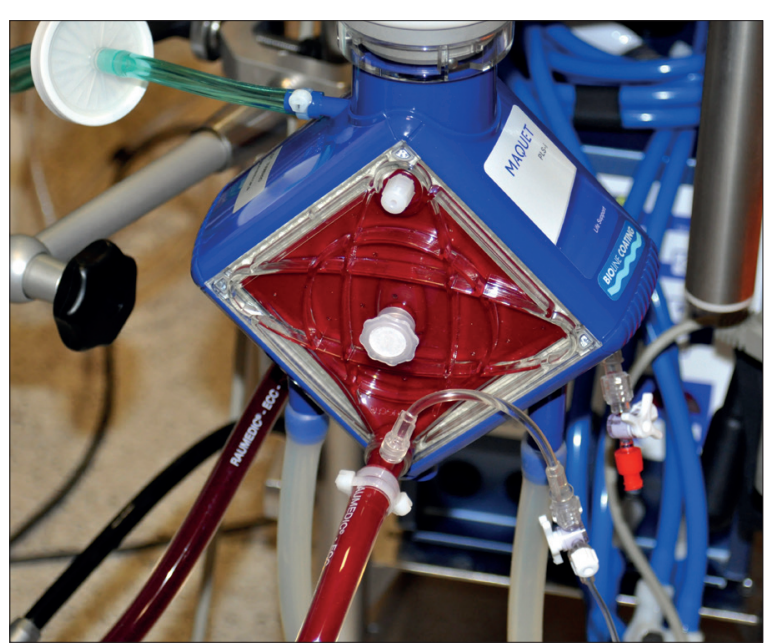

FIGURE 2. Membrane-Oxygenator of Extracorporeal Membrane Oxygenation 
ical ventilation - $\mathrm{SIMV} \mathrm{FiO}_{2}$ 0.3. Positive end-expiratory pressure (PEEP) was $6 \mathrm{~mm} \mathrm{H}_{2} \mathrm{O}$. There were numerous episodes of $V T$ and $V F$, some of which terminated spontaneously; others were defibrillated with the lowest effective energy of 50J. IABP was removed. Because of an increasing number of thrombi seen in the oxygenator, an additional unfractionated heparin infusion monitored by the aPTT was carried out. Due to elevated inflammatory parameters, namely C-reactive protein (CRP) $-17.32 \mathrm{mg} / \mathrm{dl}$; $\mathrm{N}: 0-0.5 \mathrm{mg} / \mathrm{dl}$, empirical therapy with antibiotics was introduced: Ceftriaxone + Gentamicin. The fluid balance was kept negative. Bloody secretion was present in the respiratory tract, and since the ECMO cannula ports were bleeding, they had to be treated surgically. The laboratory test findings were as follows: NT-pro BNP $6767.0 \mathrm{pg} / \mathrm{ml} ; \mathrm{N}$ : 0-125 pg/ml. Kidney and liver parameters remained normal. Total parenteral nutrition (TPN) was begun. The ECMO set was exchanged at the bedside and in sterile conditions due to clot formation in the ventricular vent. Bedside rehabilitation was carried out.

Between days 17 and 22 following CABG, the patient developed extensive bleeding from the bronchi, nose and throat. A bronchofiberoscopy, along with anterior and posterior tamponade of the nose, were carried out several times. Enteral feeding with commercial mixture through a stomach probe was introduced and TPN continued. Sedation with dexmedetomidine was introduced. As the cultures collected yielded meticilline-sensitive staphylococcus, ceftriaxon was continued. Circulation support ECMO $\mathrm{CO}$ 3.0. The inflammatory parameters were elevated - CRP was $31.4 \mathrm{mg} / \mathrm{dl}$. The laboratory findings were as follows: D-dimer $93.03 \mu / \mathrm{ml} ; \mathrm{N}$ : 0-0.5 $\mu / \mathrm{ml}$; troponin T-432.40 ng/l; N: 0-14 ng/l.

On day 38 after $C A B G$, the patient underwent a transesophageal echocardiography (TEE): the left ventricle was undilated $-52 \mathrm{~mm}$ in diastole, akinesis of the inferior wall and septum, contractility of the postero-lateral wall preserved. EF was $40-45 \%$. The right ventricle was $39 \mathrm{~mm}$ in $4 \mathrm{CH}$, while there was akinesis of the basal segment of the RV free wall, better contractility of defibrillating lead in right ventricle outflow tract (RVOT) and the apex. The patient was extubated and placed under passive oxygen therapy through a Venturi oxygen mask. In gasometry, the patient's breathing was seen to have low efficiency $\left(\mathrm{pO}_{2}-64.9 \mathrm{~mm} \mathrm{Hg}\right.$, $\mathrm{pCO}_{2}-37.6 \mathrm{~mm} \mathrm{Hg}$ ). Secretions from the bronchi had to be sucked out from time to time, while fe- ver reached $39^{\circ} \mathrm{C}$. Culture from the bronchi yielded pseudomonas aeruginosa, which led to meropenem being introduced.

On day 42 following CABG, after a follow-up TEE with preliminary heart burdening, as it was impossible to control the bleeding associated with antithrombotic therapy applied in ECMO, the patient was disconnected from ECMO, although the risk for complications was very high. The procedure was performed in an operating room. In the intraoperative period the patient had to be stabilized with catecholamines - levosimendan and dobutamine infusion. The patient was ventilated mechanically: $\mathrm{CMV} \mathrm{FiO}_{2}$ 0.7. Cardiac index $(\mathrm{Cl})$ was monitored permanently: $\mathrm{Cl}-3.6$. During the 3rd hour after the procedure, the patient was conscious with logical contact, extubated and supported with passive oxygen therapy. The patient underwent intensive breathing physiotherapy due to large secretion deposits in the bronchi and difficulties in coughing to clear the bronchi.

Between days 44 and 58 after $C A B G$, there were several episodes of respiratory failure which had to be treated with mechanical ventilation. The circulatory system was temporarily stabilized with catecholamine infusion - dobutamine and milrinon while $\mathrm{Cl}$ was 3.4. Since a fever of up to $40^{\circ} \mathrm{C}$ and elevated inflammatory parameters (CRP $25.5 \mathrm{mg} / \mathrm{dl}$ ) kept recurring, antibiotics (imipenem and colistin) were used. Once another series of microbiological test findings (including staphylococcus aureus and pseudomonas aeruginosa) were available, the treatment was changed to meronem + vancomycin followed by gentamycin, colistin and vancomycin. Although initially the patient found it difficult to swallow, oral nutrition was introduced. Intensive rehabilitation was continued, with attempts to make the patient sit up, then walk — first with support, then by himself.

On the 76th day after CABG, the patient with normal inflammatory parameters (CRP $0.9 \mathrm{mg} / \mathrm{dl}$ ), satisfactory breathing and circulation, and being conscious with logical contact, was transferred to a rehabilitation ward. In echocardiography, the left ventricle cavity was slightly dilated $-58 \mathrm{~mm}$ in diastole while the muscle thickness was normal. Akinesis of the inferior wall and interventricular septum, lateral wall contractility was preserved. EF was around $35 \%$ while LVOT VTI was $10 \mathrm{~cm}$. The left atrium was dilated. Regarding, the right ventricle, the inflow part was slightly dilated $(43 \mathrm{~mm}$ ) with very impaired 
contractility, $\mathrm{s}^{\prime} 4 \mathrm{~cm} / \mathrm{s}$, there was akinesis of the basal segment of the right ventricle free wall, with slightly better contractility of the apex. More than small IT. RVSP was around $25 \mathrm{~mm} \mathrm{Hg}$.

\section{DISCUSSION}

The paper reports the successful application of ECMO as a method of a prolonged mechanical post-CABG support in acute cardiopulmonary failure (37 days). The ECMO method provided heart muscle regeneration via temporary support of the circulatory system, which created a possibility for the heart muscle to compensate and regenerate after CABG — Figure 3. In a series of echographic examinations, EF was observed to improve from $<10 \%$ at the time before ECMO was applied to $35 \%$ at the time of discharge from the ICU. The weaning process of such patients can be very difficult, slow, and challenging. This case, to the best of our knowledge, illustrates the first reported successful veno-arterial ECMO in a patient as bridge to recovery in post-CABG cardiogenic shock associated with a dramatically poor patient condition. Owing to the fact that ECMO was applied at an early stage, no organ hyperperfusion occurred. Inotropic agents were reduced to a minimum to allow for optimal myocardial recovery while still maintaining left ventricular ejection. Due to the protective action of ECMO the patient was not exposed to large, toxic doses of catecholamines - during the whole of the follow-up, both kidney and liver parameters were normal.

Early post emergency CABG care is still a challenge for therapeutic teams. Biancari et al. [5] report- ed that in a group of 596 patients after emergency CABG, the mortality rate was around $10 \%$. In the authors' opinion, the principal independent risk factors which negatively affected the survival of post$C A B G$ patients were: increasing emergency $C A B G$ classes $(p<0.0001)$; recent myocardial infarction $(p=0.019)$; left ventricular ejection fraction $\leq 30 \%$ $(p=0.034)$; on-pump surgery $(p=0.012)$; and participating centres $(p<0.0001)$. Several studies have demonstrated mortality rates of $50 \%$ to $84 \%$ among patients requiring ECMO support after cardiothoracic surgery. Postcardiotomy cardiogenic shock occurs in approximately $1-1.5 \%$ of patients $[6,7]$. The study by Rastan et al. [7], in which the analysis comprised 517 consecutive adult patients treated with extracorporeal membrane oxygenation for refractory postcardiotomy cardiogenic shock, showed that overall hospital survival was $24.8 \%$, at a mean ejection fraction of $45.9 \% \pm 17.6 \%$ and logistic Euro-SCORE of $21.6 \% \pm 20.7 \%$. The risk factors for hospital mortality show that isolated coronary artery bypass grafting (odds ratio, 0.44 ) was protective with $34.7 \%$ hospital survival. In a study by Doll et al. [8], 219 patients who needed a postoperative ECMO support (refractory postoperative cardiogenic shock) were retrospectively analyzed. For this patient group there were substantial in-hospital (76\%) and 5 -year follow-up mortality rates (82\%). In a group of 119 patients after CABG supported by ECMO, the 30 -day mortality rate was $61 \%$. The poorest results were among patients undergoing combined CABG and aortic valve replacement with mortality rate of $95 \%$. In the study mentioned above, the independent predictors of in-hospital survival were

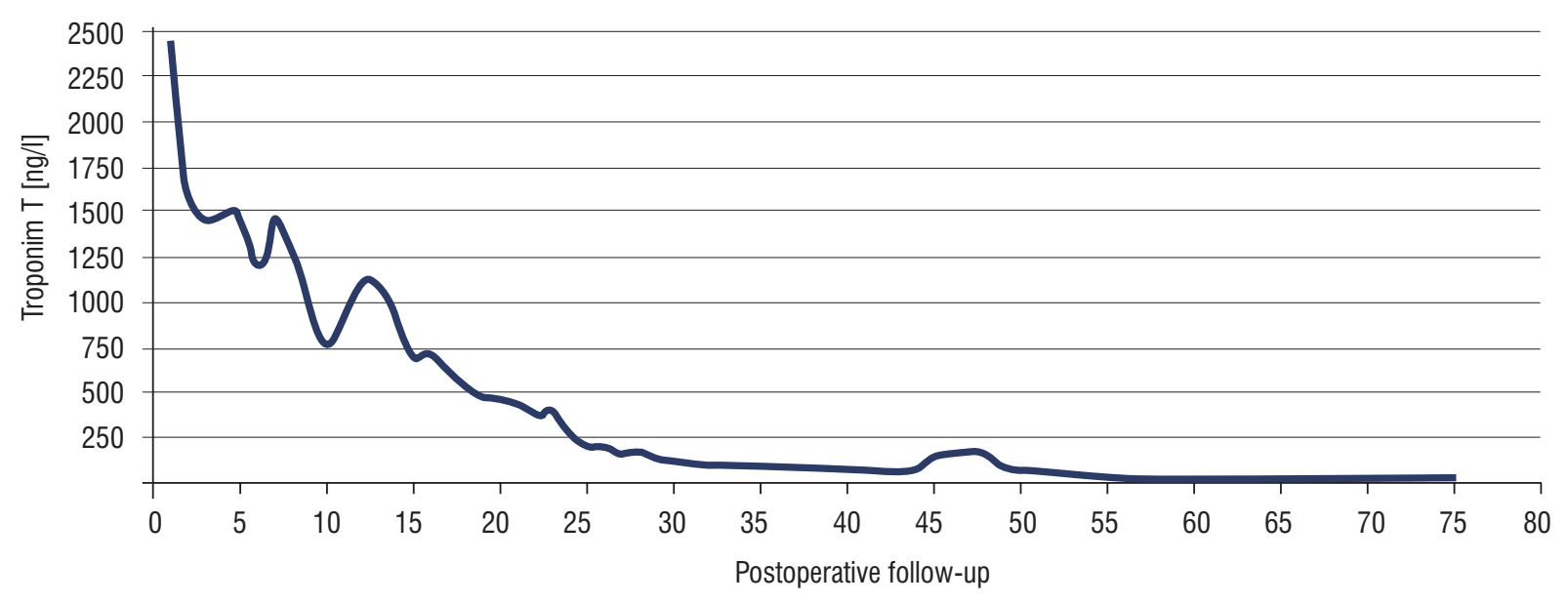

FIGURE 3. Graph depicting changes in Troponin T concentrations during the patient's postoperative follow-up 
as follows: younger age; absence of preoperative myocardial infarction; absence of diabetes; the use of an intra-aortic balloon pump; and whether the procedures performed prior to ECMO were valve, $C A B G$, or valve/CABG. In the case presented here, the patient had all of the risk factors itemized by Doll et al. This proves the presented case is unique as it points out the potential benefits resulting from a prolonged ECMO support.

The most common complications associated with ECMO include hemostasis disorders causing bleeding resulting from the necessity to apply heparin constantly [9]. The patient reported here, during his hospitalization, had 49 units of RBC concentrate transfused. This was the result of bleeding from the oral cavity and throat, which was difficult to manage. All transfused RBC preparations were leukoreduced and irradiated.

In such circumstances it is quite a challenge to maintain balanced APTT, while simultaneously preventing clot formation in the ECMO system without affecting bleeding substantially. Our patient, while undergoing ECMO therapy, was at minimally acceptable APTT times - 40-50 sec. Golding et al. [10] reported a mean transfusion rate of 53.2 of packed red blood cell units/patient and a rethoracotomy rate of $87.3 \%$ among 91 patients undergoing ECMO. In the study by Rastan et al., the mean perioperative red blood cell transfusion rate was 13 units/patient during the first 48 hours of ECMO support [7].

At present, the patient is 20 months after heart surgery, in good general condition, with no neurological complications, in NYHA class II.

\section{CONCLUSION}

The procedure can be successful only when the members of the intensive care team - physicians, paramedics, nurses, percussionists - cooperate closely.

In the centre presented here, this was the 24th procedure of ECMO used in 2014. The resultant experience makes it possible to use highly specialized medical equipment optimally, in cases whose mortality risk exceeds $90 \%$.

Conflicts of interests: The work was performed at the Department of Anaesthesiology, Institute of Cardiology, Warsaw, Poland.

The authors declare that they have no conflicts of interests. None of the authors involved in this study have any financial relationship with the parties concerned.

Source of support: No sources of financial or material support to be declared.

\section{REFERENCES}

1. McMurray JJV, Adamopoulos S, Anker SD, et al. Task Force for the Diagnosis and Treatment of Acute and Chronic Heart Failure 2012 of the European Society of Cardiology, ESC Committee for Practice Guidelines. ESC guidelines for the diagnosis and treatment of acute and chronic heart failure 2012: The Task Force for the Diagnosis and Treatment of Acute and Chronic Heart Failure 2012 of the European Society of Cardiology. Developed in collaboration with the Heart Failure Association (HFA) of the ESC. Eur J Heart Fail. 2012; 14(8): 803-869, doi: 10.1093/eurjhf/hfs105, indexed in Pubmed: 22828712.

2. Conrad $S A$, Grier $L R$, Scott $L K$, et al. Percutaneous cannulation for extracorporeal membrane oxygenation by intensivists: a retrospective single-institution case series. Crit Care Med. 2015; 43(5): 1010-1015, doi: 10.1097/CCM.0000000000000883, indexed in Pubmed: 25746749.

3. Abrams D, Combes A, Brodie D. Extracorporeal membrane oxygenation in cardiopulmonary disease in adults. J Am Coll Cardiol. 2014; 63: 2769-2778, doi: 10.1016/j.jacc.2014.03.046, indexed in Pubmed: 24814488.

4. Bartlett RH, Roloff DW, Custer JR, et al. Extracorporeal life support: the University of Michigan experience. JAMA. 2000; 283(7): 904-908, indexed in Pubmed: 10685715.

5. Biancari F, Onorati F, Rubino AS, et al. Outcome of emergency coronary artery bypass grafting. J Cardiothorac Vasc Anesth. 2015; 29(2): 275282, doi: 10.1053/j.jvca.2014.10.019, indexed in Pubmed: 25791689.

6. Smith C, Bellomo R, Raman JS, et al. An extracorporeal membrane oxygenation-based approach to cardiogenic shock in an older population. Ann Thorac Surg. 2001; 71(5): 1421-1427, indexed in Pubmed: 11383776.

7. Rastan AJ, Dege A, Mohr M, et al. Early and late outcomes of 517 consecutive adult patients treated with extracorporeal membrane oxygenation for refractory postcardiotomy cardiogenic shock. J Thorac Cardiovasc Surg. 2010; 139(2): 302-311, doi: 10.1016/j. jtcvs.2009.10.043, indexed in Pubmed: 20106393.

8. Doll N, Kiaii B, Borger M, et al. Five-year results of 219 consecutive patients treated with extracorporeal membrane oxygenation for refractory postoperative cardiogenic shock. Ann Thorac Surg. 2004; 77(1): 151-157, indexed in Pubmed: 14726052.

9. Paden ML, Conrad SA, Rycus PT, et al. ELSO Registry. Extracorporeal Life Support Organization Registry Report 2012. ASAIO J. 2013; 59(3): 202-210, doi: 10.1097/MAT.0b013e3182904a52, indexed in Pubmed: 23644605.

10. Golding LA, Crouch RD, Stewart RW, et al. Postcardiotomy centrifugal mechanical ventricular support. Ann Thorac Surg. 1992; 54(6): 1059-1063, indexed in Pubmed: 1449287. 